\title{
Caracterización de morteros mudéjares de la iglesia de San Gil Abad (Zaragoza, España): investigación de la tecnología de fabricación de morteros históricos de yeso
}

\section{Characterization of mudejar mortars from St. Gil Abbot church (Zaragoza, Spain): investigation of the manufacturing technology of ancient gypsum mortars}

\author{
J. Igea $(*)$, P. Lapuente ${ }^{(*)}$, S. Martínez-Ramírez ${ }^{(* *, * * *)}$, M. T. Blanco-Varela(***)
}

Recepción/Received: 2-XII-11

Aceptación/Accepted: 8-III-12

Publicado online/Online publishing: 13-IV-12

\section{RESUMEN}

Este trabajo se ha centrado en la investigación de la tecnología de fabricación de morteros mudéjares aplicado a la caracterización de un conjunto representativo de muestras inalteradas procedentes de una iglesia representativa de la arquitectura mudéjar aragonesa. La investigación se llevó a cabo mediante el uso combinado de técnicas analíticas incluyendo el estudio petrográfico y el análisis químico y mineralógico. Los resultados obtenidos permiten conocer la tecnología de fabricación de los morteros y clasificarlos. Todos los morteros analizados presentan una composición constante formada por una mezcla de yeso y cal, en muy baja proporción, como ligante, mientras que el árido está formado mayoritariamente por fragmentos de rocas yesíferas y carbonatadas en distinto estado de descomposición térmica. Los resultados confirman que en la fabricación de los morteros, ligante y áridos presentan la misma composición siendo estos últimos el subproducto de la misma materia prima calcinada, incorporados para elaborar el mortero tras un proceso de molienda. Esta característica ha influido positivamente en las propiedades de los morteros, mejorando su calidad.

Palabras clave: San Gil, mudéjar, mortero de yeso, caracterización, tecnología de fabricación.

\section{SUMMARY}

This work has been focused on the investigation of the technological procedure of Mudejar mortars applied to the characterization of a representative group of unaltered samples from an example church of Mudejar architecture of Aragon. The research was carried out using multi-analytical techniques including petrographic study, chemistry and mineralogical analysis. The results obtained have made it possible to classify the samples and to learn about the traditional technological procedures. All mortars analysed present a homogeneous composition. The binder is made up of a mixture of gypsum and a very low proportion of lime, while the main components of the aggregate are gypsum and carbonate rock fragments, both in a different thermal state of decomposition. The results have proved that both, binder and aggregates display the same composition in these mortars. These aggregates are the by-product of a grinding process of the previously burnt raw materials which have had a positive influence on the properties of the mortars in improving their quality.

Keywords: San Gil, mudejar, gypsum mortar, characterization, technological procedure.

(*) Universidad de Zaragoza (Zaragoza, España).

(**) Instituto de Estructura de la Materia-(IEM-CSIC) (Madrid, España).

(***) Instituto de Ciencias de la Construcción Eduardo Torroja-(IETcc-CSIC) (Madrid, España). 


\section{INTRODUCCIÓN}

Situada en el casco histórico de Zaragoza, la iglesia de San Gil Abad es un templo representativo de la arquitectura mudéjar gótica aragonesa. El monumento se localiza en un contexto urbano expuesto a condiciones ambientales adversas debido a la polución. Se construyó en el siglo XIV, con mampostería de ladrillo tomado con mortero y forma parte del Patrimonio de la Humanidad desde 2001. A lo largo de su historia, se han sucedido diversas intervenciones en el templo. La restauración más importante se llevó a cabo en época barroca, durante la cual se abrió una nueva portada en el ábside mudéjar y fueron construidas la cabecera y portada actuales del templo (1). En 2006, fueron restaurados el muro de la fachada principal, el ándito y la torre y el ábside mudéjares.

El desarrollo del arte mudéjar aragonés adquiere personalidad y autonomía propias tras la reconquista aragonesa completada a lo largo del siglo XII. El Palacio de la Aljafería podría ser citado como una de las primeras manifestaciones del mudéjar aragonés. Uno de los principales pilares a la hora de definir el arte mudéjar es el empleo en su arquitectura de tres tipos de materiales: yeso, madera y material cerámico. Aunque la unidad básica de estas edificaciones es la mampostería de ladrillo tomada con mortero, es también destacable el empleo de estructuras de madera para las cubiertas, las piezas cerámicas vidriadas en la decoración y la utilización de materiales secundarios (2). Con respecto a los morteros, han sido los de yeso o los mixtos de yeso y cal los más utilizados tanto en unión de juntas de ladrillos como en revestimientos y/o decoración de edificios.

A la hora de realizar cualquier restauración, es importante utilizar materiales y métodos que sean lo más similar posible al material al que van a sustituir. Por tanto, es necesario conocer la metodología de fabricación y diseño de los morteros históricos ya que las referencias bibliográficas acerca de las características de este tipo de materiales son muy escasas.

El principal objetivo de este trabajo es profundizar en el conocimiento de los métodos de elaboración de morteros tradicionales de yeso y cal mediante la caracterización de los morteros mudéjares de la Iglesia de San Gil Abad. Esta investigación es el primer paso dentro de un proyecto de mayor escala dedicado al estudio de distintos monumentos pertenecientes al Patrimonio Mudéjar en la región de Aragón (España) $(3,4)$, con el propósito de diseñar morteros de restauración con características compatibles a los originales.

\section{MATERIALES Y MÉTODOS}

Las muestras se tomaron en dos zonas del templo correspondientes a la arquitectura mudéjar original (Figura 1), a una altura no inferior a cinco metros para evitar

\section{INTRODUCTION}

San Gil Abbot Church, situated in the historic quarter of Zaragoza, is a representative temple belonging to Aragonese Gothic Mudejar architecture. The monument is located in an urban environment and suffers the unfavourable conditions of pollution. It was built in the $14^{\text {th }}$ century with brick masonry and mortar-filled joints and has been part of Human Heritage since 2001. Throughout history, the monument has undergone a considerable number of transformations. The most important being a restoration made during the Baroque period, with a new portal opened in the Mudejar apse and the present head and façade of the temple were constructed (1). In 2006, the main façade, external corridor and the Mudejar tower and apse were restored.

The Aragonese Mudejar style was developed in the $12^{\text {th }}$ century after the Reconquest of Aragon territories were completed. One of the first manifestations of Aragonese Mudejar could be the Aljafería Palace. Mudejar architecture is defined by the use of three kinds of materials: gypsum, wood and ceramic materials. This art is characterised by the use of brick masonry with mortarfilled joints as the main materials. In addition a very diverse group of materials were employed; wood structures in the roofs, ornamental glazed ceramics and the use of other secondary materials (2). With regard to the mortars, gypsum and gypsum-lime mixed mortars were the most common materials employed for filling joints, in tilework and other decorations.

In the restoration works, it is very important to note that both, new and old materials display similar characteristics. Therefore, the need exists to know the manufacturing technology of ancient gypsum-lime mortars. However, previous information is very scarce about the characteristics of these kinds of mortars.

The main objective of this paper is to understand of ancient gypsum-lime mortar mixing methods through the characterization of the Mudejar mortars of St. Gil Abbot Church. This investigation is the first step of a large-scale proyect dedicated to the study of different monuments from the Mudejar Heritage in the region of Aragon (Spain) $(3,4)$, with the purpose of designing restoration mortars with compatible characteristics.

\section{MATERIALS AND METHODS}

Samples were taken from the upper part of two zones of the building identified as original Mudejar architecture (Figure 1), above a minimum of five metres high, in 


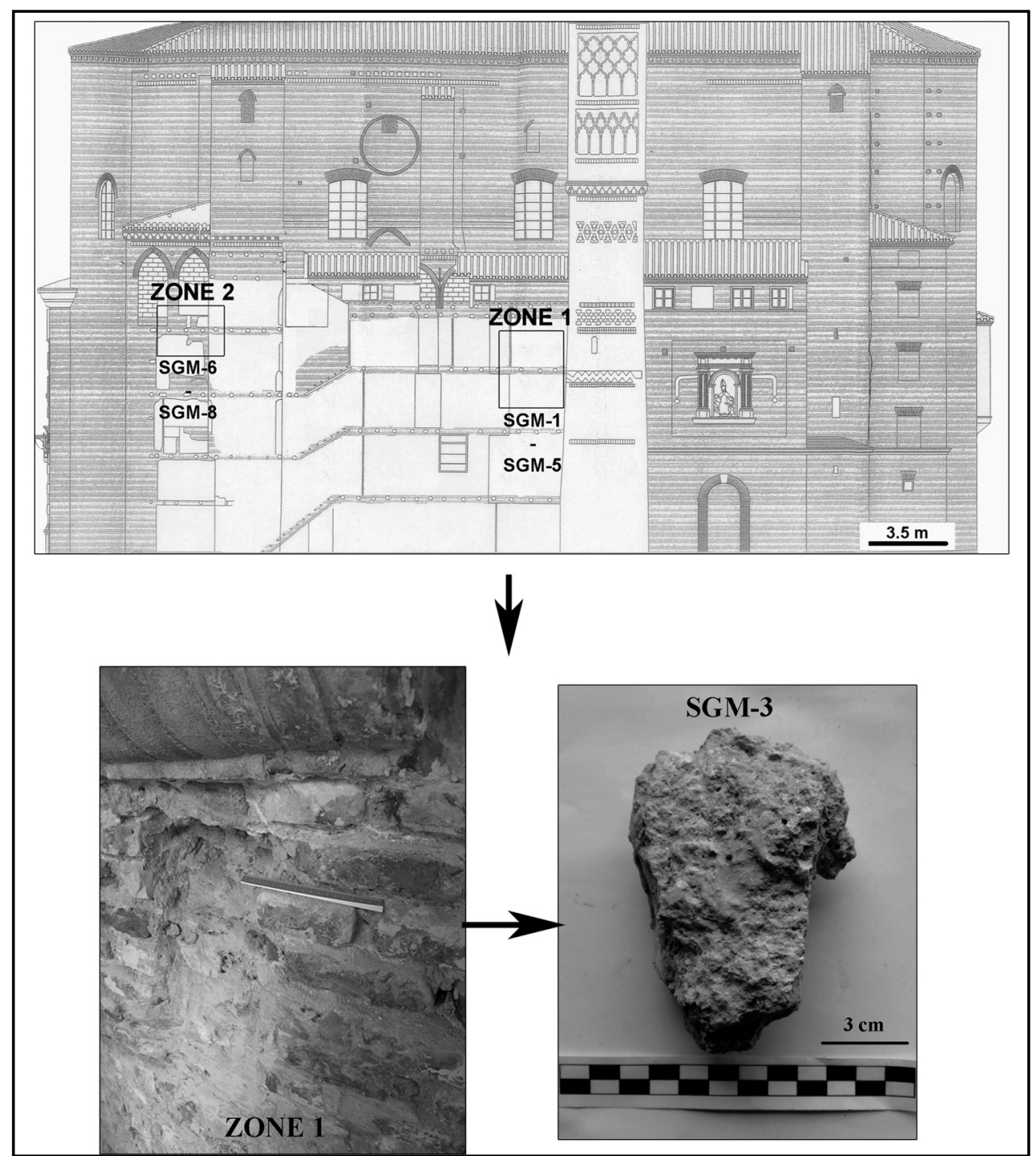

Figura 1. Plano de la iglesia de San Gil Abad mostrando las áreas de muestreo (arriba). Zona 1 de muestreo junto con una muestra del mortero de juntas (abajo).

Figure 1. Plan of St. Gil Abbot Church showing the sampling areas (above). Sample zone 1 together with a joint mortar specimen (below).

los procesos de succión capilar del agua del subsuelo. Se seleccionó un conjunto representativo de ocho muestras inalteradas tomadas tanto en la parte externa como interna de los morteros de junta originales. Las muestras fueron examinadas macroscópicamente mediante lupa binocular.

El estudio petrográfico-mineralógico se llevó a cabo con un microscopio óptico de polarización (MO) modelo Olympus AX70, con equipo de microfotografía. Las láminas delgadas se elaboraron con direcciones de corte perpendiculares a los bordes de los fragmentos con el fin de observar posibles modificaciones composicionales y texturales desde el borde externo, al borde interno de los morteros. Además, se les aplicó una tinción mixta con rojo de alizarina para la distinción entre los carbonatos, calcita y dolomita. El análisis petrográfico de morteros order to avoid any phenomena caused by rising damp. A representative group of eight unaltered samples were collected from both, external and internal parts of the original joint mortars and were examined macroscopically using a binocular microscope.

The petrographic and mineralogical study was carried out using an Olympus AX70 optical microscope (OM) with an integrated digital camera. Thin sections were prepared according to perpendicular directions from the fragment boundaries with the purpose of observing compositional and textural variations between both external and internal parts of the mortars. Thin sections were Alizarin-stained to help distinguish between calcite and dolomite. The petrographic analysis of historic mortars allows identifying the nature of the different 
históricos permite identificar la naturaleza de las distintas especies minerales que constituyen el mortero incluyendo la composición y el estado de cristalinidad del aglomerante. Igualmente, con el microscopio óptico se realiza el análisis textural estableciéndose la distribución del tamaño de grano, la morfología o la mineralogía del árido y la estimación de la dosificación ligante / árido (5). En esta investigación, la cuantificación de las proporciones de ligante y árido se llevó a cabo mediante un tratamiento digital de imágenes a partir de microfotografías representativas de las láminas delgadas, en condiciones de luz polarizada plana y cruzada. El software utilizado (ADImag 1.2) fue desarrollado por el Servicio Científico-Técnico de la Universidad de Zaragoza. Una revisión de la metodología empleada puede encontrarse en la bibliografía (6).

Es preciso indicar que cada muestra fue analizada de forma íntegra, sin separar sus componentes principales (ligante y árido), mediante la combinación de distintas técnicas analíticas (FTIR, DRX, ATD-TG). Previamente, las muestras se tamizaron $(<63 \mu \mathrm{m})$, tras su reducción de tamaño de partícula en un mortero de ágata, mezclando fragmentos del centro y del borde externo, para evitar diferencias relativas a la profundidad de carbonatación.

La espectroscopía de infrarrojo por transformada de Fourier (FTIR) se usó para la identificación cualitativa de los componentes principales y de los posibles productos de reacción que pueden formarse en las pastas. Se utilizó un espectroscopio de infrarrojo modelo Nicolet 6700 de doble haz, de doble red de difracción, con una resolución espectral de $4 \mathrm{~cm}^{-1}$ y posibilidad de registro entre 4.000 y $400 \mathrm{~cm}^{-1}$. La caracterización químico-mineralógica cualitativa se completó con la difracción de rayos-X (DRX), mediante el método del polvo cristalino desorientado. Las muestras se analizaron con un difractómetro Phillips XPert MPD Pro utilizando monocromador de grafito y rendija automática de divergencia con radiación de $\mathrm{Cu} K \alpha(\lambda=1,5406 \AA)$, que trabaja a $40 \mathrm{kV}$ y $30 \mathrm{~mA}$ en un tubo de rayos- $X$, en barridos con paso angular de $0.02(2 \theta)$ y 0.5 segundos de tiempo de paso y velocidad de exploración de $15 \%$ min.

Los métodos térmicos empleados en este estudio fueron el análisis termogravimétrico (ATG) y el análisis térmico diferencial (ATD) que permiten realizar determinaciones cuantitativas de los distintos componentes en la muestra total. Para el análisis mediante ATD-TG, se utilizó un equipo TA SDT Q600 que opera en condiciones de atmósfera de nitrógeno. La velocidad de calentamiento fue de $4^{\circ} \mathrm{C} /$ minuto para el tramo desde la temperatura ambiente hasta $250{ }^{\circ} \mathrm{C}$ y de $10^{\circ} \mathrm{C} / \mathrm{minu}-$ to hasta el final del experimento. mineralogical mortar phases including the composition and crystalline state of the binder. Also it makes it possible to carry out textural analysis to establish the particle size distribution (grading), shape and mineralogy of the aggregate and estimate the binder/aggregate ratios (5). In this paper, the quantification of the binder and aggregate proportions were carried out using digital imaging processing from representative microphotographs of thin sections taken in both light conditions (crossed and plane polarized). The software used (ADImag 1.2) was developed by the Technical and Scientific Services of Zaragoza University; see (6) for an overview of the methodology involved.

Worthy of note is that each sample was studied as a whole (matrix and aggregate) through the different analytical techniques (FTIR, XRD and DTA-TG). They were previously milled in agate mortar down to $<63 \mu \mathrm{m}$ particle size, mixing fragments from the inner and outer parts of specimens, to avoid differences related to carbonation depth.

Fourier-transformed infrared spectroscopy (FTIR) was used for the qualitative identification of the main mineralogical components and the hypothetical reacting products which can form in the mixtures. Nicolet 6700 infrared spectroscopy equipped with double haze, double diffraction net, $4 \mathrm{~cm}^{-1}$ spectral resolution was employed and FTIR spectra were collected in the range $4000-400 \mathrm{~cm}^{-1}$. The qualitative mineralogical and chemical characterization was completed with $X$-ray diffraction (XRD) using a powder crystalline method in bulk samples. Powder samples were analyzed with a Philips XPert MPD Pro diffractometer using graphite monocromator and automatic slit, with $\mathrm{Cu} K \alpha$ radiation $(\lambda=1.5406 \AA)$, working $40 \mathrm{kV}$ and $30 \mathrm{~mA}$ in an $X$-ray

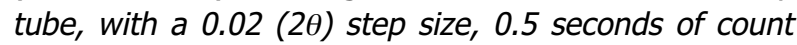
time per step and $15 \%$ min of exploration velocity.

Thermal methods employed in this work were thermogravimetric (TG) and differential thermal analysis (DTA) in order to make quantitative determinations of different components in the total sample. DTA-TG analysis was recorded in a nitrogen atmosphere with a TA SDT Q600 mixed-analyser. The heating was programmed with an initial temperature rise from ambient to $250^{\circ} \mathrm{C}$, at a rate of $4^{\circ} \mathrm{C} / \mathrm{min}$; a second rise was applied at $10 \mathrm{o} / \mathrm{min}$ until the end of the experiment. 


\section{RESULTADOS Y DISCUSIÓN}

\subsection{Descripción de las muestras}

Los materiales seleccionados corresponden a morteros que presentan un espesor variable entre $15-75 \mathrm{~mm}$ recogidos entre hiladas de ladrillos con un grueso que oscila entre 40-60 mm (Figura 1). Las observaciones macroscópicas revelan que todos los morteros de junta presentan elevada cohesión ligante/árido y que no muestran evidencias de alteración. Las muestras están formadas principalmente por una mezcla de yeso y áridos de tamaño variado, con tonalidad blanquecina-grisácea. Se distinguen como áridos, fragmentos de rocas carbonatadas y agregados de yeso blanquecino. En muy escasa proporción se reconocen partículas oscuras de ceniza y fragmentos cerámicos angulosos de color anaranjado (Tabla 1). Ocasionalmente, el árido de tamaño grueso puede superar los $5 \mathrm{~mm}$ de diámetro. También se detecta la presencia de abundante macroporosidad.

\subsection{Caracterización petrográfica}

Los resultados obtenidos a partir de la caracterización petrográfica bajo el microscopio óptico de polarización (MO) y el análisis digital de imágenes, se resumen en la Tabla 2. Los morteros presentan una matriz de tamaño de grano fino (microcristalina) con un árido que tiene una amplia distribución de tamaño de grano aunque destacan los áridos gruesos $(0.5 \mathrm{~mm}->2 \mathrm{~mm})$, formados preferentemente por fragmentos líticos. El árido de tamaño de grano fino $(0,016 \mathrm{~mm}-0,25 \mathrm{~mm})$ está compuesto a su vez por fragmentos líticos, aunque también se han identificado distintos clastos monocristalinos (Tabla 2). La estimación de la dosificación ligante/árido es muy diferente de unos morteros a otros y varía entre 1,5:1 y 3,5:1 (Tabla 2).

\section{RESULTS AND DISCUSSION}

\subsection{Description of samples}

The thickness of the mortars analysed range between 15 and $75 \mathrm{~mm}$. These samples were collected from different brick courses varying from 40 to $60 \mathrm{~mm}$ (Figure 1). From a macroscopic point of view, all joint mortars displayed high binder/aggregate cohesion and no evidence of alteration was observed. At first sight, samples consisted chiefly of a mixture of gypsum and aggregates with an inhomogeneous grain-size distribution and white-greyish colour. Different kinds of materials could be recognized among the aggregates such as carbonate rock fragments or whitish gypsum aggregates with black charcoal particles and orange angular shaped ceramic fragments in very low proportions (Table 1). Occasionally, coarse aggregates exceeded $5 \mathrm{~mm}$ in diameter. Relatively abundant macroporosity was also identified.

\subsection{Petrographic characterization}

The petrographic characterization by means of OM study and the results obtained through digital image analyses are summarised in Table 2. The mortars showed a finegrained binder while aggregates displayed a wide rangesize distribution prevailing the coarse-grain fraction $(0.5$ $\mathrm{mm}->2 \mathrm{~mm}$ ), mainly consisting of lithic fragments. In the fine-grain fraction $(0.016 \mathrm{~mm}-0.25 \mathrm{~mm})$, lithic fragments and different monocrystalline grains were also recognized (Table 2). The estimated values for the binder/aggregate ratio are very distinct for samples and range between 1.5:1 and 3.5:1 (Table 2).

Tabla 1 / Table 1

Características macroscópicas y medida del color de las muestras de mortero estudiadas. Macroscopic characteristics and colour measurement of the mortars studied.

\begin{tabular}{|c|c|c|c|c|}
\hline $\begin{array}{l}\text { Muestras I } \\
\text { Samples }\end{array}$ & $\begin{array}{l}\text { Área de muestreo / } \\
\text { Sampling area }\end{array}$ & Altura / Height (m) & $\begin{array}{l}\text { Color (Obs. visual) I } \\
\text { Colour (Visual obs.) }\end{array}$ & $\begin{array}{l}\text { Presencia de áridos I } \\
\text { Presence of Aggregates }\end{array}$ \\
\hline SGM-1 & \multirow{4}{*}{$\begin{array}{l}\text { NE. Mampostería fachada / NE. } \\
\text { Masonry façade }\end{array}$} & \multirow{4}{*}{$5 \mathrm{~m}$} & \multirow{8}{*}{$\begin{array}{l}\text { Blanco-grisáceo / } \\
\text { White-greyish }\end{array}$} & \multirow{8}{*}{$\begin{array}{l}\text { Fragmentos roca carbonatada / } \\
\text { Carbonate rock fragments } \\
\text { Fragmentos roca yesífera / } \\
\text { Gypsum rock fragments } \\
\text { Partículas de ceniza / Charcoal } \\
\text { particles } \\
\text { Fragmentos cerámicos / } \\
\text { Ceramic fragments }\end{array}$} \\
\hline SGM-2 & & & & \\
\hline SGM-3 & & & & \\
\hline SGM-4 & & & & \\
\hline SGM-5 & $\begin{array}{l}\text { NE. Repisa mampostería fachada / } \\
\text { NE. Ledge from masonry façade }\end{array}$ & $7 \mathrm{~m}$ & & \\
\hline SGM-6 & $\begin{array}{l}\text { NE. Ándito fachada / } \\
\text { NE. Corridor façade }\end{array}$ & $10 \mathrm{~m}$ & & \\
\hline SGM-7 & \multirow{2}{*}{$\begin{array}{l}\text { NE. Ándito fachada / } \\
\text { NE. Corridor façade }\end{array}$} & \multirow{2}{*}{$10 \mathrm{~m}$} & & \\
\hline SGM-8 & & & & \\
\hline
\end{tabular}


Tabla 2 / Table 2

Resumen de los resultados obtenidos a partir de la caracterización petrográfica mediante microscopía óptica y tratamiento digital de imágenes. Se expone la cuantificación en las muestras de distintas características texturales tanto del ligante como del árido (distribución de tamaño del árido, macroposidad del ligante y dosificación ligante/árido), así como la mineralogía de los componentes principales.

Summary of the petrographic characterization by means of Optical Microscopy and the results obtained through digital image processing. Binder and aggregate texture (aggregate size distribution, binder macroporosity and binder/aggregate ratio) and the main mineralogical components of the aggregate were quantified in the samples.

\begin{tabular}{|c|c|c|c|c|c|c|c|c|c|}
\hline & \multicolumn{8}{|c|}{ Muestras / Samples } \\
\hline & & SGM-1 & SGM-2 & SGM-3 & SGM-4 & SGM-5 & SGM-6 & SGM-7 & SGM-8 \\
\hline \multicolumn{2}{|c|}{$\begin{array}{l}\text { Ligante Yeso-Cal (\%) I } \\
\text { Gypsum-Lime Binder (\%) }\end{array}$} & 60 & 75 & 60 & 75 & 78 & 72 & 60 & 75 \\
\hline \multicolumn{2}{|c|}{ Macroporosidad / Macroporosity } & MA & A & A & MA & MA & MB & MA & $M$ \\
\hline \multicolumn{2}{|c|}{$\begin{array}{c}\text { Dosificación Ligante/Árido / } \\
\text { Binder/Aggregate Ratio }\end{array}$} & $1.5: 1$ & $3: 1$ & $1.5: 1$ & 3:1 & $3.5: 1$ & $2.5: 1$ & $1.5: 1$ & $3: 1$ \\
\hline \multicolumn{2}{|c|}{ Árido (\%) / Aggregate (\%) } & 40 & 25 & 40 & 25 & 22 & 28 & 40 & 25 \\
\hline \multirow{4}{*}{$\begin{array}{l}\text { Árido grueso / } \\
\text { Coarse aggregates } \\
(0.5 \mathrm{~mm}->2 \mathrm{~mm}) \\
\text { Fragmentos líticos / } \\
\text { Lithic Fragments }\end{array}$} & Yeso / Gypsum & 10 & 7.5 & 8 & 6 & 5.5 & 9.5 & 17 & 6 \\
\hline & Carbonato / Carbonate & 11 & 8.5 & 10.5 & 12 & 4.5 & 7.5 & 6.5 & 7.5 \\
\hline & $\begin{array}{l}\text { Yeso + Carbonato / } \\
\text { Gypsum + Carbonate }\end{array}$ & 15 & 3.5 & 12 & 2.5 & 5.5 & 6 & 9 & 8.5 \\
\hline & $\begin{array}{c}\text { Rocas silicatadas (Cuarcita, } \\
\text { Arenita, Lutita) / Silicate } \\
\text { rocks (Quartzite, Sandstone, } \\
\text { Mudstone }\end{array}$ & 1.5 & 2.5 & 5.5 & 3 & 4 & 2.5 & 5 & 1 \\
\hline \multirow{2}{*}{$\begin{array}{c}\text { Árido fino / } \\
\text { Fine aggregates } \\
(0.016 \mathrm{~mm}->0.25 \mathrm{~mm})\end{array}$} & $\begin{array}{l}\text { Cuarzo + Feldespatos / } \\
\text { Quartz + Feldspars }\end{array}$ & 1 & 0.5 & 2.5 & 1 & 0.5 & 1.5 & 1 & 1 \\
\hline & $\begin{array}{l}\text { Fragmentos Líticos / } \\
\text { Lithic Fragments }\end{array}$ & 1 & 2.5 & 1.5 & 0.5 & 1.5 & 0.5 & 1.5 & 0.5 \\
\hline $\begin{array}{l}\text { Accesorios I } \\
\text { Accessories }\end{array}$ & $\begin{array}{l}\text { Partículas de ceniza y } \\
\text { fragmentos cerámicos / } \\
\text { Charcoal particles and } \\
\text { Ceramic fragments }\end{array}$ & 0.5 & - & - & - & 0.5 & 0.5 & - & 0.5 \\
\hline
\end{tabular}

Leyenda / Legend: A = Alta / High, MA = Media-Alta / Medium-High, M = Media / Medium, MB = Media-Baja / Medium-Low.

La macroporosidad observada en el ligante presenta morfologías preferentemente subredondeadas (Figura 2A). Este tipo de porosidad esférica se ha relacionado (7) con mezclas de aditivos de naturaleza orgánica. Asimismo, el estudio petrográfico ha confirmado que los morteros analizados presentan una fuerte unión ligante-árido y ausencia de microfisuras y de precipitación de sales en la porosidad.

El ligante está formado principalmente por yeso con tamaño de grano fino y bajas proporciones de cal. La calcita, que se encuentra dispersa en la matriz de las morteros, procede de la carbonatación de la cal apagada y se caracteriza por presentar un tamaño de grano muy fino (micrita $<4 \mu \mathrm{m}$ ). Además, como resultado de una incompleta reacción de carbonatación del ligante, se ha identificado cal viva tanto distribuida de forma homogénea como concentrada en zonas específicas de la matriz de algunas muestras (Figura 2B), no descartando que una carbonatación deficiente ocasionara carbonatos amorfos. En zonas puntuales, son visibles bordes de
Binder macroporosity of mostly sub-rounded shapes was distinguished (Figure 2A). This kind of spherical porosity has been related (7) to organic additives. In addition, petrographic study revealed that the mortars analysed displayed a strong binder-aggregate bond and an absence of microfractures and salts precipitation in the porosity.

Fine-grained gypsum is the main component of the binder with a very low proportion of lime. In most cases the matrix is partially made up of very finely crystallised calcite (micrite $<4 \mu \mathrm{m}$ ) derived from the carbonation of the slaked lime. Occasionally, pure lime can be seen distributed both homogeneously and concentrated in specific areas of the matrix (Figure 2B), as a result of incomplete carbonation reaction in the binder. At this stage amorphous carbonates might also have been formed. Reaction rims are sometimes visible at the interface between aggregates and the binder caused by a differential carbonation reaction carried out in the 

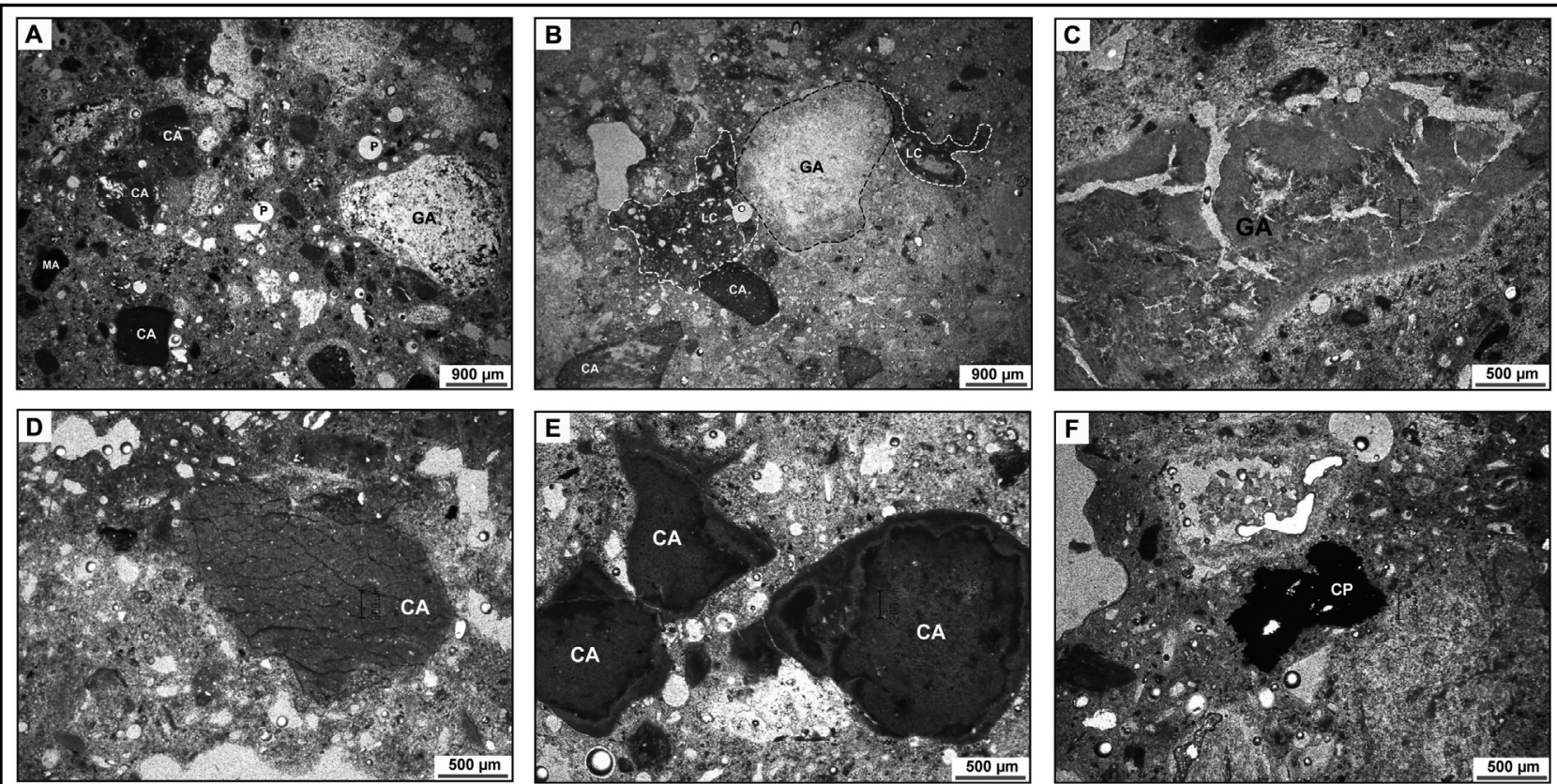

Leyenda / Legend

GA: Árido yesífero / Gypsum aggregate

CA: Árido carbonatado / Carbonate aggregate

MA: Árido lutítico / Mudstone aggregate

P: Poro / Pore

LC: Concentración de cal / Lime concentration

CP: Partícula de ceniza / Charcoal particle

Figura 2. Microfotografías de las muestras tomadas bajo MO (nícoles paralelos). A) Aspecto característico de los morteros analizados.

B) Detalle de la concentración de cal en zonas específicas de la matriz. C) Árido yesífero que presenta fisuración-agrietamiento por

contracción térmica durante la cocción. D) Fragmento de roca carbonatada parcialmente descompuesto con presencia de

fisuración-agrietamiento por contracción térmica originada durante el proceso de calcinación. E) Áridos carbonatados calcinados presentando aureolas de reacción en los bordes por hidratación diferencial de la cal. F) Detalle de una partícula de ceniza.

Figure 2. Microphotographs of the samples taken under OM (parallel nicols). A) Characteristic appearance of the mortars analysed. B)

Detail of lime concentrated in specific areas of the matrix. C) Gypsum aggregate displaying shrinkage-cracking derived from the

burning process. D) Partly burnt carbonate fragment showing shrinkage-cracking derived from the calcination process. E) Overburnt carbonate fragments displaying boundary reactions caused of differential lime hydration. F) Detail of charcoal particle.

reacción en la interfase matriz-árido, como consecuencia de la carbonatación diferencial del ligante. Finalmente, tapizando la porosidad ha sido identificada también precipitación de calcita secundaria (microsparita 4-20 $\mu \mathrm{m}$ ).

La naturaleza de los áridos inorgánicos es variada, pero mayoritariamente están constituidos por fragmentos de rocas yesíferas, carbonatadas $y$, en menor medida, de composición silicatada (Tabla 2), con un tamaño de grano grueso. Estas observaciones son acordes a las realizadas en otros trabajos sobre morteros históricos (8-10). Los áridos yesíferos son de tipo alabastrino y presentan distintos grados de descomposición térmica. Se observan de forma conjunta granos que no han sido afectados por la cocción (incocidos) con otros que presentan fisuración y agrietamiento por contracción térmica (sobrecocidos) (Figura 2C). Estos agregados pueden contener inclusiones microcristalinas de anhidrita además de impurezas lutítico-margosas. Este tipo de inclusiones son habituales matrix. Secondary calcite (microsparite 4-20 $\mu \mathrm{m}$ ) precipitation can also be seen in the porosity of the matrix.

The nature of the inorganic aggregates is very diverse but consists mainly of coarse-grained gypsum, carbonate and scarce silicate rock fragments (Table 2), as with other works (8-10). The gypsum aggregates are of alabaster type at different stages of thermal decomposition. Some grains which were unaffected during the burning process (underburnt) have been observed together with others displaying shrinkagecracking (overburnt) (Figure 2C). In these sorts of aggregates, anhydrite microcrystalline inclusions can be seen as well as mud-marly impurities. These inclusions are all common in the alabaster nodules derived from the sedimentary sequences of the tertiary Ebro basin. In agreement with previous studies on other Mudejar 
en los nódulos alabastrinos que proceden de las secuencias sedimentarias de la cuenca terciaria del Ebro. De acuerdo con trabajos previos realizados en distintos monumentos mudéjares (11), se han diferenciado cuatro tipos de árido yesífero distintos, atendiendo a sus características ópticas, texturales-microestructurales y composicionales. A estos cabe añadir la presencia de cantidades abundantes de fragmentos de yeso impuro con abundante matriz de carbonato micrítico y minerales arcillosos (Tabla 2).

En cuanto a los áridos carbonatados, presentan una composición de calcita micrítica e inclusiones minoritarias de cuarzo. En algunas de estas muestras se han reconocido proporciones escasas de fragmentos de roca dolomítica. Una característica destacable es que en todas las muestras han sido observados fragmentos de rocas carbonatadas en diferente estado de descomposición térmica: desde fragmentos de roca carbonatada sin transformar, a otros parcialmente descompuestos (Figura 2D) e incluso fragmentos carbonatados calcinados que en ocasiones muestran aureolas de reacción por hidratación diferencial de la cal en los bordes (Figura 2E).

De forma adicional, pueden distinguirse una gran variedad de componentes (Tabla 2): fragmentos de rocas silicatadas (cuarcitas, arenitas y lutitas) así como inclusiones de cuarzo monocristalino y feldespatos con tamaño de grano fino, todos ellos en muy bajas proporciones. Además, se distinguen partículas carbonosas de origen vegetal en la mayor parte de las muestras (Figura 2F), que podrían ser atribuidas a impurezas derivadas del horno de cocción. Finalmente, el análisis mediante MO ha permitido identificar en algunos morteros y con carácter accesorio, fragmentos angulosos de piezas cerámicas con idénticas características petrográficas que las de los ladrillos empleados en la edificación del templo.

Las características texturales de los áridos principales (fragmentos de rocas yesíferas y carbonatadas), tales como su amplio rango de distribución de tamaños y sobre todo la morfología extremadamente angulosa de los áridos de tamaño de grano grueso (Figura 2E), evidencian que estos agregados fueron previamente machacados.

Así, el análisis textural ha revelado que tanto los áridos yesíferos como los carbonatados son el subproducto de la molienda de las correspondientes rocas usadas como materia prima para la elaboración del ligante y que fueron incorporados posteriormente de forma intencionada a la pasta. La diferenciación de distintos tipos de árido de naturaleza yesífera, confirma que los morteros analizados están compuestos por un yeso multifásico producido artesanalmente por la calcinación desigual de aljez (12). El proceso de cocción del yeso proporcionó productos heterogéneos: desde bloques de yeso crudo sin alterar durante monuments (11), four types of gypsum aggregates can be distinguished by optical, textural, micro-structural and compositional characteristics. In addition, high proportions of impure gypsum fragments can also be observed with a matrix consisting of abundant carbonate micrite and clay minerals (Table 2).

Carbonate aggregates based on micritic calcite and scarce quartz inclusions were also identified. Low proportions of dolomite rock fragments were found in several samples. It must be noted that in all samples, carbonate rock fragments can also be observed at different stages of thermal decomposition: underburnt, partly burnt (Figure 2D) and overburnt fragments which can show boundary reactions caused by differential lime hydration (Figure 2E).

In addition, a very diverse group of components can be identified (Table 2): silicate rock fragments (quartzite, mudstones and sandstone) as well as fine-grained quartz and feldspar grains appear in very low proportions. Also, charcoal particles identified in the majority of the samples (Figure 2F) could be attributed to impurities from the kiln fuel. Finally, microscopic analysis evidenced that several mortars included rare ceramic fragments with the same petrographic characteristics as the bricks used in the building construction.

Concerning the textural analysis, the wide range-size distribution should be pointed out together with the extremely irregular angular shapes of the large size aggregates of both gypsum and carbonate rock fragments (Figure 2E).

Both aspects show that these aggregates had been previously crushed. In fact, textural analysis has proved that both, gypsum and carbonate aggregates, were a byproduct of the grinding process of each previously burnt raw material and were deliberately added to the mortars. The presence of a variety of gypsum aggregates show that the mortars analysed were the result of a multi-phase structure of the gypsum, obtained by craft production methods (12). The burnt gypsum process provided heterogeneous products: from underburnt gypsum blocks, which were not affected during the 
el proceso de cocción, a yeso con distinto grado de descomposición térmica, sin llegar en ningún caso a la calcinación intensa con formación de cal libre.

Asimismo, la detección de cal junto a la identificación de fragmentos de rocas carbonatadas aducen la utilización de materia prima calcárea previamente calcinada en la fabricación de los morteros $(13,14)$. Estos áridos carbonatados presentan también distinto grado de descomposición térmica: desde fragmentos de roca que no se han visto afectados por la cocción a nódulos de cal mostrando aureolas de reacción debidas a las diferencias en el proceso de hidratación de la cal.

\subsection{Estudio mineralógico}

En la Tabla 3 y en la Figura 3 se exponen los resultados del análisis cualitativo de las fases mineralógicas identificadas mediante FTIR. Todos los morteros presentan idéntica asociación mineralógica compuesta por yesocuarzo-calcita-dolomita y como impureza nitratos. La elevada absorbancia de la señal debida al yeso indica claramente que es un componente del mortero y no una impureza originada por el ataque externo de sulfatos como se ha descrito en otros materiales del Patrimonio (15-17). En general, las frecuencias a las que aparecen las diferentes bandas de absorción se corresponden bien con las citadas en la bibliografía $(18,19)$. El espectro se ha dividido en 4 zonas, a partir de intervalos de frecuencias de absorción definidos (Tabla 3).

El principal componente de los morteros es el yeso, identificado a partir de fuertes bandas de absorción (3.550$3.405,1.620,1.144-1.120 \mathrm{~cm}^{-1}$ ) (Figura 3). En todos los espectros, se detecta una fuerte banda en torno a 1440 $\mathrm{cm}^{-1}$ causada por la presencia de carbonatos. También se identifica el cuarzo en todas las muestras mediante distintas bandas de absorción (798-778, 462 cm-1). Asimismo, el burning process, to blocks in different thermal decomposition stages but without reaching the limit of the burning process with a free CaO formation.

Moreover, the lime detection and the identification of carbonate rock fragments, confirm that previously burnt raw carbonate rocks were used during the manufacture of the mortars $(13,14)$. Carbonate aggregates also show different degrees of thermal decomposition: rock fragments can be observed which were not affected during the burning process (underburnt) and overburnt fragments showing boundary reactions caused by differential hydration of lime.

\subsection{Mineralogical study}

Table 3 and Figure 3 show the results obtained through qualitative analysis of the mineralogical phases identified by means of FTIR. All mortars displayed the same mineralogical association composed of gypsum-quartzcalcite-dolomite and nitrates as an impurity. The high intensity of the absorbance band from gypsum indicates that calcium sulphate is a mortar binder. In previous works (15-17) it was determined that gypsum presence in building materials can also becomes from external sulphate attack. In general, the frequencies at which the different absorption bands appear are consistent with those given in the bibliography $(18,19)$. Spectra have been divided into 4 zones through defined absorption frequency intervals (Table 3).

Gypsum is the main component of mortars and is identified by its strong absorption bands (3550-3405, 1620, 1144-1120 cm-1) (Figure 3). In all spectra, a strong band shown at $1440 \mathrm{~cm}^{-1}$ is attributed to the presence of carbonates. Different absorption bands related to quartz were also detected in all samples (798778, $\left.462 \mathrm{~cm}^{-1}\right)$. Finally, potassium nitrate can be

Tabla 3 / Table 3

Fases mineralógicas y sus respectivas bandas de absorción características identificadas mediante FTIR. Mineralogical phases and corresponding absorption bands identified by means of FTIR.

\begin{tabular}{|c|c|c|c|c|c|}
\hline \multirow{2}{*}{$\begin{array}{l}\text { Componente mineral / } \\
\text { Mineral Compound }\end{array}$} & \multicolumn{5}{|c|}{ Posición de las bandas de absorción / Position of Absorption Bands $\left(\mathrm{cm}^{-1}\right)$} \\
\hline & $\left(4000-2000 \mathrm{~cm}^{-1}\right)$ & $\left(2000-1200 \mathrm{~cm}^{-1}\right)$ & $\left(1200-1000 \mathrm{~cm}^{-1}\right)$ & & \\
\hline Yeso / Gypsum & $\begin{array}{l}3550 \mathrm{~F} \\
3405 \mathrm{~F} \\
3245 \mathrm{D}\end{array}$ & $\begin{array}{l}1685 \mathrm{M} \\
1620 \mathrm{~F}\end{array}$ & $\begin{array}{l}1144 \mathrm{~F} \\
1120 \mathrm{~F} \\
1005 \mathrm{H}\end{array}$ & \multicolumn{2}{|c|}{$\begin{array}{l}670 M \\
602 M\end{array}$} \\
\hline Cuarzo / Quartz & - & - & - & \multicolumn{2}{|c|}{$\begin{array}{l}798 \mathrm{M} \\
778 \mathrm{M} \\
462 \mathrm{~F}\end{array}$} \\
\hline Calcita / Calcite & - & \multirow{2}{*}{$\sim 1440 \mathrm{~F}$} & \multirow{2}{*}{-} & \multirow{2}{*}{875 M } & $713 \mathrm{D}$ \\
\hline Dolomita / Dolomite & - & & & & $729 \mathrm{D}$ \\
\hline $\begin{array}{l}\text { Nitrato potásico / } \\
\text { Potassium Nitrate }\end{array}$ & - & $1384 \mathrm{D}$ & - & & \\
\hline
\end{tabular}

Leyenda / Legend: F = Fuerte / Strong, M = Media / Medium, D = Débil / Weak, H = Hombro / Shoulder. 
nitrato potásico puede estar presente como impureza en los morteros. En algunos espectros se registra una absorción aguda a $1.384 \mathrm{~cm}^{-1}$, que se correlaciona con la zona de vibración del grupo $\mathrm{NO}_{3}{ }^{-}$(Figura 3). Este componente suele estar presente como producto de degradación en los materiales de construcción de monumentos localizados en áreas urbanas (20).

La caracterización mineralógica de las muestras mediante DRX se resume en la Tabla 4. Se expresan también los poderes reflectantes empleados para el análisis semicuantitativo de cada una de las fases mineralógicas cristalinas detectadas. Estos resultados corroboran las observaciones realizadas tanto al MO como por FTIR. Los resultados de DRX confirman que la fase mineralógica displayed as an impurity in the mortars. In several spectra, a sharp band at $1384 \mathrm{~cm}^{-1}$ was registered which is correlated to the $\left(\mathrm{NO}_{3}\right)^{-}$vibration zone (Figure 3). Nitrates are typical degradation products found on monuments located in polluted areas (20).

The mineralogical characterization by means of XRD of these samples is summarised in Table 4. Reference intensity ratios were used to carry out semi-quantitative analysis of the detected crystalline mineralogical phases. Results agree with the OM and FTIR data. The $X R D$ patterns indicate gypsum as the main mineralogical phase in all mortars. As observed in Table 4, low

Tabla 4 / Table 4

Fases mineralógicas identificadas en cada uno de los morteros y análisis semicuantitativo a partir del método de los poderes reflectantes.

Mineralogical phases identified in each mortar and semi-quantitative analysis through reference intensity ratios.

\begin{tabular}{|c|c|c|c|c|c|c|c|}
\hline $\begin{array}{c}\text { Muestras I } \\
\text { Samples }\end{array}$ & Phy $\left(I_{10 \AA} \times 1\right)$ & $\mathrm{Gp}\left(I_{7.6 A} \times 1.5\right)$ & Anh $\left(I_{3.49 A} \times 1.5\right)$ & Qtz $\left(I_{3.34 \AA} \times 1.5\right)$ & $\operatorname{Pg}\left(I_{3.17 \AA} \times 0.97\right)$ & Cal $\left(I_{3.03 A} \times 1\right)$ & $\operatorname{Dol}\left(I_{2.88 A} \times 1\right)$ \\
\hline SGM-1 & I & +++ & I & + & I & + & + \\
\hline SGM-2 & - & +++ & + & + & + & I & - \\
\hline SGM-3 & I & +++ & I & + & + & ++ & I \\
\hline SGM-4 & I & +++ & I & + & + & + & + \\
\hline SGM-5 & I & +++ & + & + & + & I & - \\
\hline SGM-6 & I & +++ & + & + & I & ++ & + \\
\hline SGM-7 & - & +++ & + & ++ & + & I & - \\
\hline SGM-8 & - & +++ & + & + & + & ++ & I \\
\hline
\end{tabular}

Leyenda / Legend: Phy = Filosilicatos (illita-moscovita) / Phyllosilicates (illite-moscovite); Gp = Yeso / Gypsum; Anh = Anhidrita / Anhydrite; Qtz = Cuarzo / Quartz; Pg = Plagioclasa / Plagioclase; Cal = Calcita / Calcite; Dol = Dolomita / Dolomite. Proporciones relativas / Relative proportions: +++: mayoritario / majority; ++: abundante / abundant, +: escaso / scarce; I: indicio / trace; -: ausente / absent.

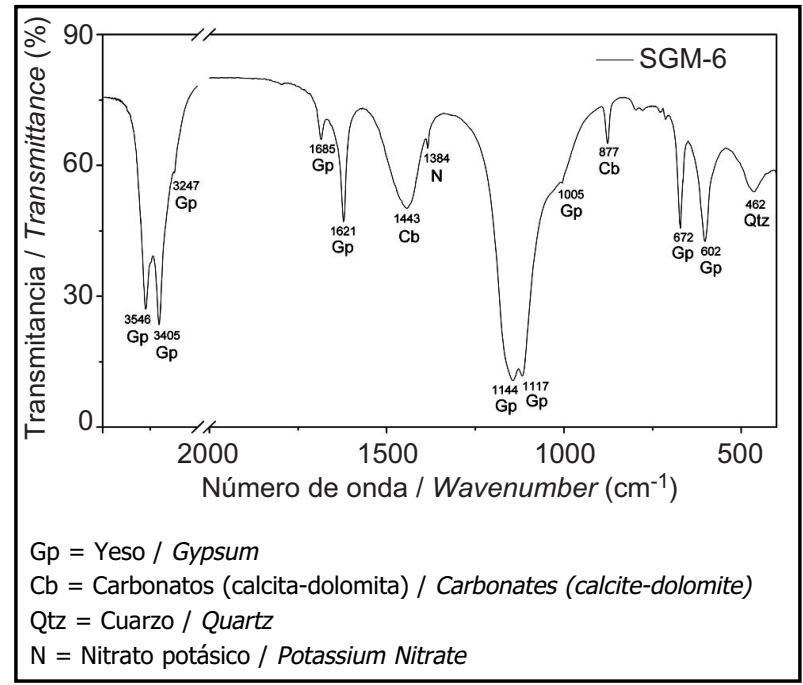

Figura 3. Espectro FTIR y componentes identificados entre 4000-400 $\mathrm{cm}^{-1}$ correspondientes a la muestra SGM-6.

Figure 3. FTIR spectra and compounds identified from 4000 to $400 \mathrm{~cm}^{-1}$ in the sample SGM-6.

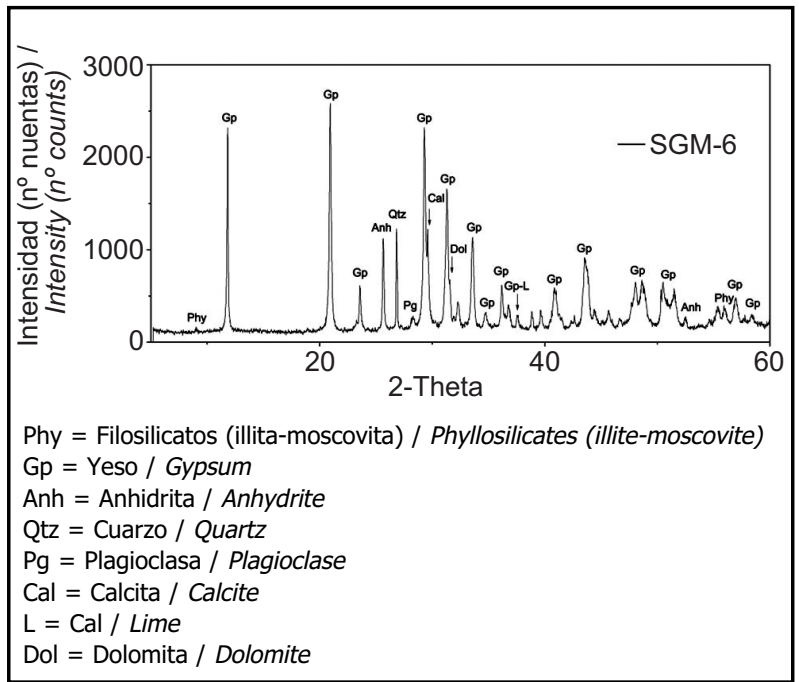

Figura 4. Difractograma de la muestra SGM-6. Figure 4. Diffractogram of the sample SGM-6. 
predominante en todos los morteros es el yeso. Como puede observarse en la Tabla 4, también han sido detectadas proporciones menores de calcita, cuarzo, anhidrita, feldespatos (plagioclasa) y filosilicatos de tipo illitamoscovita, mientras que de forma muy minoritaria ha sido identificada dolomita.

Las diferencias establecidas en la cuantificación de calcita y dolomita mediante las distintas técnicas mineralógicas (Tablas 3 y 4) pueden estar motivadas por tres razones principalmente:

- La ausencia de dolomita puede ser debida a su menor proporción relativa en los morteros, como se ha descrito en la caracterización al MO.

- Las reflexiones de difracción de estos componentes se ven enmascaradas por las reflexiones del yeso.

- Se han formado fases carbonatadas con bajo grado de cristalinidad durante el fraguado del mortero.

Del mismo modo, la detección de cal $(2,40 \AA)$ resulta compleja ya que se ve enmascarada por el yeso (Figura 4). Sin embargo, la presencia de ambos componentes ha quedado corroborada a partir de los estudios de MO.

\subsection{Análisis térmicos}

Los resultados de los análisis de ATD-TG se muestran en la Tabla 5 y en la Figura 5. Todos los morteros tienen un comportamiento térmico similar con curvas en las que se observan al menos cuatro efectos endotérmicos. La pérdida total de peso varía entre 22 y $24 \%$, indicando la homogeneidad en la composición de los morteros. proportions of calcite, quartz, anhydrite, feldspars (anorthite) and phyllosilicates (illite-moscovite) were also detected while those of dolomite were extremely scarce.

Three main reasons explain the differences estimated in the quantification of carbonate phases (calcite and dolomite) through the different mineralogical techniques used (Tables 3, 4):

- No identification of dolomite may be due to its extreme scarcity as described in the OM characterization.

- The diffraction reflections of these compounds are masked by those of the gypsum.

- Poorly crystalline carbonates were formed during the binder setting.

The identification of pure lime (2.40 $\AA$ ) was made difficult through being masked by the gypsum (Figure 4). However, the existence of both components was confirmed with OM studies.

\subsection{Thermal analysis}

The results derived from the DTA-TG analysis are shown in both Table 5 and Figure 5. All mortars display similar thermal behaviour with curves in which at least four endothermic effects can be observed. The total weight loss ranges from 22 to $24 \%$, indicating the homogeneity of the mortars composition.

Tabla 5 / Table 5

Se exponen las transformaciones térmicas en las curvas de ATD/TG: pérdida de peso (\%) en cada rango de temperatura $\left({ }^{\circ} \mathrm{C}\right)$, la cuantificación del agua estructural $\left(\mathrm{H}_{2} \mathrm{O}_{\text {Est }}\right)$, yeso $\left(\mathrm{CaSO}_{4} \times 2 \mathrm{H}_{2} \mathrm{O}\right)$, calcita $\left(\mathrm{CaCO}_{3}\right)$ y dolomita $\left[\mathrm{CaMg}\left(\mathrm{CO}_{3}\right)_{2}\right]$ en las muestras analizadas, así como las temperaturas correspondientes a la transformación de anhidrita (Anh), la presencia de materia orgánica (MO) y la descomposición del $\mathrm{CaCO}_{3}$.

Thermal transformations in DTA-TG curves are summarised: weight loss (\%) percentage at each temperature $\left({ }^{\circ} \mathrm{C}\right)$ range and bound water $\left(\mathrm{H}_{2} \mathrm{O}_{E s t}\right)$, gypsum $\left(\mathrm{CaSO}_{4} \times 2 \mathrm{H}_{2} \mathrm{O}\right)$, calcite $\left(\mathrm{CaCO}_{3}\right)$ and dolomite $\left[\mathrm{CaMg}\left(\mathrm{CO}_{3}\right)_{2}\right]$ quantification in the samples analysed are shown.

The phase change in anhydrite (Anh), the presence of organic matter (MO) and $\mathrm{CaCO}_{3}$ decomposition temperatures are also given.

\begin{tabular}{|c|c|c|c|c|c|c|c|c|c|c|c|c|c|c|}
\hline \multirow{3}{*}{$\begin{array}{c}\text { Muestras I } \\
\text { Samples }\end{array}$} & \multicolumn{7}{|c|}{$\begin{array}{l}\text { Pérdida de peso }(\%) \text { en cada rango de temperatura }\left({ }^{\circ} \mathrm{C}\right) / \\
\text { Weight loss }(\%) \text { per temperature range }\left({ }^{\circ} \mathrm{C}\right)\end{array}$} & \multirow{3}{*}{$\begin{array}{c}\% \\
\mathrm{H}_{2} \mathrm{O}_{\text {Est }}\end{array}$} & \multirow{3}{*}{$\begin{array}{c}\% \\
\text { Yeso I } \\
\text { Gypsum }\end{array}$} & \multirow{3}{*}{$\begin{array}{c}\% \text { Calci- } \\
\text { ta / Cal- } \\
\text { cite }\end{array}$} & \multirow{3}{*}{$\begin{array}{c}\% \\
\text { Dolomita I } \\
\text { Dolomite }\end{array}$} & \multirow{3}{*}{$\begin{array}{c}\mathrm{T}_{\text {Anhlll }} \rightarrow \\
\text { Anhll } \\
\left({ }^{\circ} \mathrm{C}\right)\end{array}$} & \multirow{3}{*}{$\begin{array}{l}\mathrm{T}_{\mathrm{MO}} \\
\left({ }^{\circ} \mathrm{C}\right)\end{array}$} & \multirow{3}{*}{$\begin{array}{l}\mathrm{T}_{\mathrm{CO} 2} \\
\left({ }^{\circ} \mathrm{C}\right)\end{array}$} \\
\hline & \multirow{2}{*}{$25-100$} & \multirow{2}{*}{$100-150$} & \multirow{2}{*}{$150-560$} & \multicolumn{2}{|c|}{$560-710$} & \multirow{2}{*}{$710-1000$} & \multirow{2}{*}{\begin{tabular}{|c|} 
Pérdida \\
total $/$ \\
Total \\
loss (\%)
\end{tabular}} & & & & & & & \\
\hline & & & & $560-590$ & $590-710$ & & & & & & & & & \\
\hline$S G M-1$ & 0.79 & 13.75 & 2.4 & \multicolumn{2}{|c|}{4.97} & 2.02 & 23.93 & 2.4 & 65.7 & 11.3 & - & 257 & 454 & 694 \\
\hline SGM-2 & 1.09 & 15.04 & 2.33 & \multicolumn{2}{|c|}{2.03} & 2.05 & 22.54 & 2.33 & 71.9 & 4.6 & - & 258 & 447 & 662 \\
\hline$S G M-3$ & 1.07 & 13.35 & 2.98 & \multicolumn{2}{|c|}{4.42} & 0.59 & 22.41 & 2.98 & $\begin{array}{ll}63.8 \\
\end{array}$ & 10 & - & 258 & 442 & $672-694$ \\
\hline SGM-4 & 1.08 & 14.82 & 2.58 & \multicolumn{2}{|c|}{3.33} & 0.66 & 22.47 & 2.58 & 70.8 & 7.6 & - & 258 & 445 & 683 \\
\hline$S G M-6$ & 1.13 & 12.95 & 1.79 & \multicolumn{2}{|c|}{4.44} & 1.89 & 22.2 & 1.79 & 61.9 & 10.1 & - & 248 & 433 & 672 \\
\hline SGM-8 & 0.8 & 14.83 & 3.72 & 0.82 & 1.47 & 1.43 & 23.07 & 3.72 & 70.9 & 3.3 & 1.7 & 259 & - & $576-669$ \\
\hline
\end{tabular}


El yeso es el principal componente de las muestras analizadas, atendiendo a su contenido relativo (62-72\%), obtenido a partir de los valores de TG (Tabla 5). Estos resultados corroboran los derivados del estudio mediante las distintas técnicas analíticas. Los componentes carbonatados (calcita y/o dolomita) se encuentran en los morteros en muy bajas proporciones (5-11\%). La proporción de dolomita es relativamente menor que la de calcita de acuerdo con el análisis de DRX.

En la mayor parte de las muestras, la descomposición de los carbonatos en las curvas ATD aparece de forma continua, sin mostrar estadios térmicos. Por tanto, los picos endotérmicos de calcita y dolomita aparecen solapados, complicando la identificación de dolomita. Este hecho, unido a que no hay evidencias mediante la observación bajo MO de la presencia de esta fase mineral en la matriz de los morteros, permite la asignación de dolomita como componente minoritario únicamente del árido de los morteros.

Ambos carbonatos (calcita y dolomita) han sido discriminados únicamente en la muestra SGM-8 (Figura 5B) mediante la distinción de dos picos endotérmicos (21); la pérdida de peso en el intervalo de 560-590 ${ }^{\circ} \mathrm{C}$ estaría causada por la descomposición de dolomita, mientras que la pérdida de peso en el rango entre $590-710^{\circ} \mathrm{C}$, se asocia a la descarbonatación de calcita. En algunos morteros la pérdida de $\mathrm{CO}_{2}$ puede producirse en dos pasos, a $672{ }^{\circ} \mathrm{C}$ y $694^{\circ} \mathrm{C}$ respectivamente (Figura $5 \mathrm{~A}$ ), lo que justificaría la presencia de carbonatos con un origen diferente (primario y secundario) o de carbonatos amorfos sin cristalinidad (22).
Gypsum is the main component in the samples studied according to the approximate contents (62-72\%) obtained through TG values (Table 5). These data agree with results obtained by means of different analytical techniques. Carbonate compounds (calcite and/or dolomite) are found in mortars in very low proportions (5-11\%). Dolomite proportion is lower than that of the calcite according to XRD analysis.

In the majority of the samples, carbonates decomposition in the DTA curves seems to occur in a continuous form, without thermal steps; so the endothermic peaks of the different carbonates (calcite and dolomite) appear overlapped, making the identification of dolomite difficult. This circumstance, together with no evidence of dolomite in the paste under the OM study, has allowed the dolomite assignation as a minority component of the aggregates.

Both carbonates (calcite and dolomite) have been differentiated only in the SGM-8 sample (Figure 5B) by means of the distinction of two endothermic peaks (21); the weight loss within the range of $560-590^{\circ} \mathrm{C}$ is due to the decarbonation of magnesium carbonate, while the weight loss in the range of $590-710^{\circ} \mathrm{C}$ is associated to the decarbonation of calcium carbonate. In several mortars, the double signal at $672{ }^{\circ} \mathrm{C}$ and $694^{\circ} \mathrm{C}$ (Figure $5 A$ ) attributed to the loss of $\mathrm{CO}_{2}$, could be due to different carbonates (primary and secondary) or the presence of an amorphous one (22).

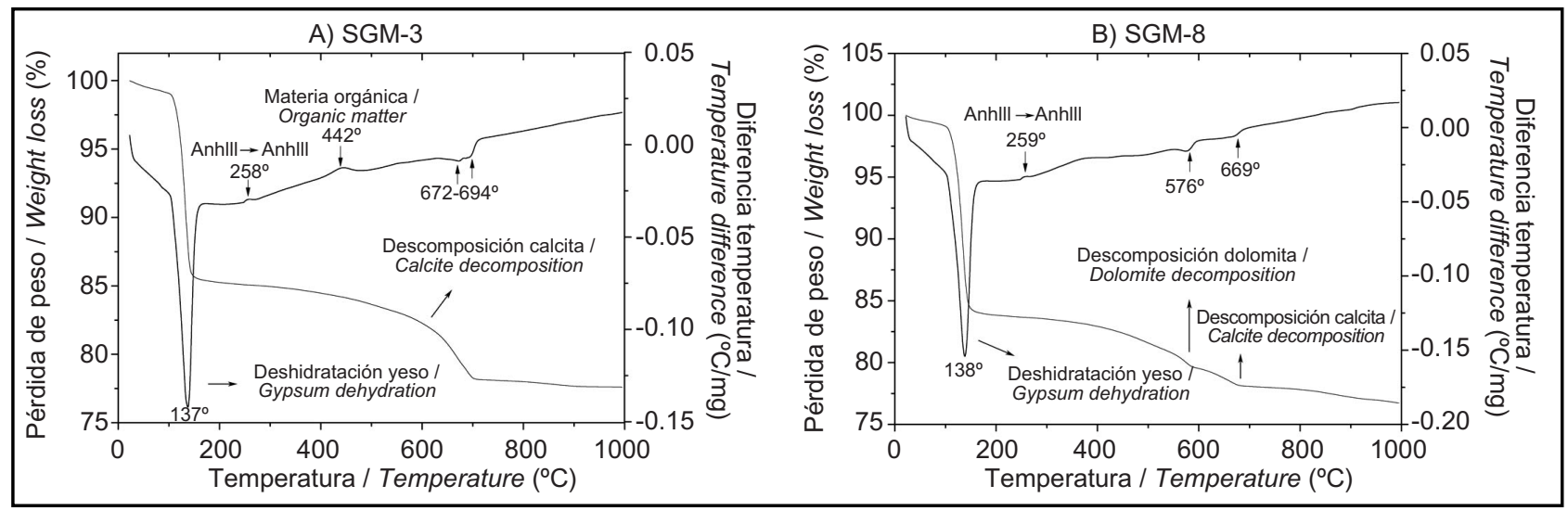

Figura 5. Curvas de ATD-TG correspondientes a las muestras SGM-3 y SGM-8 respectivamente, con sus picos característicos: Deshidratación del yeso, descomposición de los carbonatos, transformación de anhidrita (Anh) y presencia de materia orgánica. A) Puede observarse el efecto endotérmico doble $\left(672-694^{\circ} \mathrm{C}\right)$ causado por la descomposición de la calcita. B) Se distinguen dos picos endotérmicos atribuidos a carbonatos; dolomita $\left(576^{\circ} \mathrm{C}\right)$ y calcita $\left(669^{\circ} \mathrm{C}\right)$.

Figure 5. Characteristic peaks are shown in the DTA-TG curves corresponding to SGM-3 and SGM-8 samples: gypsum dehydration, carbonates decomposition, the phase change in anhydrite (Anh) and the presence of organic matter. A) A double endothermic effect (672-694 $\left.{ }^{\circ} \mathrm{C}\right)$ can be seen caused by the decomposition of calcite. B) Two endothermic peaks can be distinguished attributed to carbonates; dolomite $\left(576^{\circ} \mathrm{C}\right)$ and calcite $\left(669^{\circ} \mathrm{C}\right)$. 
En la Figura 5 pueden observarse, a su vez, las transformaciones térmicas a partir del análisis de ATD. Las muestras presentan al menos dos picos exotérmicos; el pico exotérmico agudo que se encuentra en torno a los $250{ }^{\circ} \mathrm{C}$ podría corresponder al comienzo de la transformación cristalográfica de la anhidrita III en anhidrita II, mientras que el segundo se debe a reacciones exotérmicas producidas en los morteros entre $300-500{ }^{\circ} \mathrm{C}$, que son atribuidas a la deshidroxilación de componentes arcillosos o a la presencia de materia orgánica (23).

\section{CONCLUSIONES}

La caracterización de los morteros de unión de estilo mudéjar a través de la combinación de distintas técnicas analíticas ha permitido clasificar los materiales analizados y realizar una serie de consideraciones acerca de la tecnología de fabricación de estos morteros históricos.

Todas las muestras estudiadas presentan una composición homogénea con variaciones texturales claramente visibles. Así, los valores de dosificación ligante/árido estimados a partir del tratamiento digital de imágenes son muy diferentes y varían entre $1,5: 1$ y 3,5:1.

El ligante se compone mayoritariamente de yeso al cual han sido adicionadas proporciones minoritarias de cal. La composición del árido está constituida principalmente por fragmentos de rocas yesíferas y carbonatadas en distinto estado de descomposición térmica. Algunos autores (12) indican la dificultad a la hora de clasificar estos materiales como pastas o morteros dentro de los conceptos actuales, debido a la presencia de material yesífero con granulometría variada.

Además, se distinguen fragmentos de rocas silicatadas (cuarcitas, arenitas y lutitas) en muy bajas proporciones. Cuarzo y feldespatos son también componentes accesorios del árido. Además, la presencia en muy baja proporción de filosilicatos así como de materia orgánica, ha quedado evidenciada por las distintas técnicas analíticas empleadas. Exceptuando las partículas carbonosas, el resto de áridos identificados son frecuentes en los depósitos sedimentarios de la cuenca del Ebro, de donde pudo extraerse la materia prima para la elaboración de los morteros.

La fabricación de morteros artesanales de yeso con proporciones variables de cal es una constante en la edificación a partir del siglo VIII, tras la invasión árabe de la Península Ibérica (24). La adición de cal a los morteros mejora la trabajabilidad de la pasta retardando el fraguado del yeso. Además incrementa la resistencia mecánica a largo plazo de los materiales de construcción, debido al proceso de carbonatación de la cal $(25,26)$.
Thermal transformations can also be observed in Figure 5 through DTA analysis. The samples show at least two exothermic events; a sharp exothermic peak at around $250{ }^{\circ} \mathrm{C}$ could correspond to the beginning of the transition of anhydrite III to anhydrite II, whereas exothermic reactions produced in mortars in the range 300-500 ${ }^{\circ} \mathrm{C}$ attributed to either the dehydroxilation of clay compounds or the presence of organic matter in the mortars according to (23).

\section{CONCLUSIONS}

The samples characterization by means of the combination of different analytical techniques has made it possible to classify the materials being examined and also to understand the technological processes involved in the make up of these ancient mortars.

All the samples studied present a homogeneous composition while the textural variations between mortars were evidenced. The estimated values for the binder/aggregate ratio through digital image analysis are very distinct for samples and range between 1.5:1 and 3.5:1.

The binder consists mainly of gypsum to which lime, in very low proportions, was added. Gypsum and carbonate rock fragments in different thermal decomposition stages are the main component of the aggregates. According to (12), these materials can not be readily defined as what are known today as pastes or mortars due to the presence of gypsum materials with different grain size.

In addition, silicate rock fragments (quartzite, mudstones and sandstone) appear in very low proportions. Quartz and feldspar are also accessory aggregates. Furthermore, the presence in very low proportions of phyllosilicates as well as organic matter has been corroborated by means of the different analytical techniques used. With the exception of charcoal fragments, the remainder of the aggregates is normally derived from sedimentary deposits from the Ebro basin used as raw materials in the manufacture of the mortars.

Ancient mortars manufactured with gypsum and fairly low proportions of lime have been very common in building construction since the $8^{\text {th }}$ century after the Arabian invasion of the Iberian Peninsula (24). The addition of lime to mortars improves binder workability and slows hardening of the gypsum. Moreover, it increases the mechanical strength of the construction materials in the long term due to the carbonation lime process $(25,26)$. 
El uso intencionado de los subproductos de la molienda de ambas materias primas para ser utilizados como áridos ha influido positivamente en las propiedades de los morteros permitiendo un buen estado de conservación a lo largo del tiempo.

\section{AGRADECIMIENTOS}

Esta investigación ha sido financiada por el Departamento de Patrimonio del Gobierno de Aragón y por el Programa Europa de la Caja de Ahorros de la Inmaculada, asi como por el Ministerio de Educación y Ciencia (Proyecto CONSOLIDER CSD2007-00058) y el Gobierno Regional de Madrid ( Programa Geomateriales). Los autores del trabajo agradecen los permisos concedidos para el estudio del material seleccionado.
On the other hand, the deliberate addition of byproducts from the grinding process as aggregates in the mixtures of both raw materials influenced positively the properties of the mortars that allow their conservation over time.

\section{ACKNOWLEDGMENTS}

This research was financially supported by Departamento de Patrimonio del Gobierno de Aragón and the Europe Programme from Caja de Ahorros de la Inmaculada and by the Spanish Ministry of Education and Science (Project CONSOLIDER CSD2007-00058) and the Regional Government of Madrid (Geomaterials Programme). We are grateful for the permission to study the selected material.

\section{BIBLIOGRAFÍA / BIBLIOGRAPHY}

(1) García de Paso, A.: La Iglesia Parroquial de San Gil Abad de Zaragoza. Ed: Institución Fernando el Católico. Zaragoza (1985).

(2) Borrás, G.: Arte mudéjar aragonés. Eds: Caja de Ahorros y Monte de Piedad de Zaragoza, Aragón y Rioja y Colegio Oficial de Arquitectos Técnicos y Aparejadores de Zaragoza. Zaragoza (1985).

(3) Igea, J.: Caracterización de los materiales de construcción del Mudéjar aragonés. Diseño de nuevos morteros para su aplicación en restauración. Tesis Doctoral, Universidad de Zaragoza (España) (2011).

(4) Igea, J.; Lapuente, P.; Blanco-Varela, M. T.; Martínez-Ramírez, S.: "Ancient gypsum mortars from Sta. María Magdalena church (Zaragoza, Spain): Advances in technological manufacture". Proceedings of 2 Historic Mortars Conference, edited by J. Válek, C. Groot, J. J. Hughes. Prague (2010), pp. 197-205.

(5) Álvarez Galindo, J. I.; Ontiveros, E.: Morteros. Programa de normalización de estudios previos aplicado a bienes inmuebles. Junta de Andalucía, Consejería de Cultura e Instituto Andaluz del Patrimonio Histórico (eds.), Sevilla. PH Cuadernos, vol. 19 (2006), pp. $92-$ 146.

(6) Carò, F.; Di Giulio, A.: "Reliability of textural analysis of ancient plasters and mortars through automated image analysis", Mater. Charact., vol. 53, no 2-4 (2004), pp. 243-257. doi: 10.1016/j.matchar.2004.06.014.

(7) Elsen, J.: "Microscopy of historic mortars-a review", Cem. Concr. Res., vol. 36 (2006), pp. 1416-1424. doi: 10.1016/j.cemconres.2005.12.006.

(8) Luxán, M. P.; Dorrego, F.; Laborde, A.: "Ancient gypsum mortars from S. Engracia (Zaragoza, Spain): Characterization. Identification of additives and treatments". Cem. Concr. Res., vol. 25 (1995), pp. 1755-1765. doi: 10.1016/0008-8846(95)00171-9

(9) Franzoni, E.; Sandrolini, F.; Baldazzi, L.: Characterization of gypsum-selenite plasters from historic buildings in the Emilia-Romagna region (Italy). Proceedings of $2^{\text {th }}$ Historic Mortars Conference edited by Válek, J.; Groot, C.; Hughes. J. J., Prague (2010), pp. 157-164.

(10) Silva Hermo, B.; Prieto Lamas, B.; Rivas Brea, T.; Pereira Pardo, L.: "Gypsum-induced decay in granite monuments in Northwestern Spain" Mater. Construcc., vol. 60, no 297, (2010), pp. 97-110.

(11) Igea, J.; Lapuente, P.; Mandado, J.: "Aproximación al Estudio Petrográfico de Áridos Yesíferos en Morteros. Ejemplo de Sta. María de la Huerta, Magallón (Zaragoza)". Macla, vol. 9 (2008), pp. 133-134.

(12) Bustamante, R.; Sánchez de Rojas, M. I.: "Study of plaster finishes on San Pedro de los Francos church at Calatayud". Mater. Construcc., vol. 57, no 286 (2007), pp. 53-64.

(13) Hughes, J.; Leslie, A. B.: "The petrography of lime inclusions in historic lime based mortars". Proceedings of $8^{\text {th }}$ Euroseminar on Microscopy Applied to Building Materials. Athens (2001), pp. 359-364.

(14) Zamba, I. C.; Stamatakis, M .G.; Cooper, F. A.; Themelis, P. G.; Zambas, C. G.: "Characterization of mortars used for the construction of Saithidai Heroon Podium (1 $1^{\text {st }}$ century AD) in ancient Messene, Peloponnesus, Greece". Mater. Charact., vol. 58 (2007) $1229-1239$. doi: 10.1016/j.matchar.2007.07.004.

(15) Durán A.; Robador, M. D.; Jiménez de Haro, M. C.; Herrera, L K.; Gimena, P.; Pérez-Rodríguez, J. L.: "Seville City Hall Chapter Room ceiling decoration". Mater. Construcc., vol. 60, no 267 (2010), pp. 83-95. 
(16) Carmona-Quiroga, P. M.; Panas, I.; Svensson, J. E.; Johansson, L. G.; Blanco-Varela, M.T.; Martínez-Ramírez, S.: "Protective performances of two anti-graffiti treatments towards sulfite and sulfate formation in SO2 polluted model environment". Applied Surface Science 257 (2010), pp. 852-856.

(17) Blanco-Varela, M. T.; Aguilera, J.; Martínez-Ramírez, S.; Palomo, A.; Sabbioni, C.; Riontino, C.; Zappia, G.;Van Valen, K.; Toumbakari, E .E.: "Thaumasite formation in hydraulic mortars by atmospheric $\mathrm{SO}_{2}$ deposition". Mater Construcc, vol. 51, no 263-264 (2001) pp.109-125. (18) De la Cruz, I.; Vázquez, T.; Fernández Peña, O.: "IR spectroscopy of sulphates in clinkers and cements". Mater. Construcc., vol. 36, no 201 (1986), pp. 25-42.

(19) Gadsden, J. A.: Infrared spectra of minerals and related inorganic compounds. Ed: The Butterworth Group, London (1975).

(20) Rampazzi, L.; Bugini, R.: "St. Lorenzo Basilica in Milan: Integral approach to characterisation of historical mortars". e-preservation science, vol. 3 (2006), pp. 21-26.

(21) Álvarez Galindo, J. I.; Navarro, I.; García Casado, P. J.: "Thermal, mineralogical and chemical studies of the mortars used in the cathedral of Pamlona (Spain)". Thermochim. Acta, vol. 365 (2000), pp. 177-187. doi: 10.1016/S0040-6031(00)00624-9.

(22) Montoya, C.; Lanas, J.; Arandigoyen, M.; Navarro, I.; García Casado, P. J.; Álvarez, J.I.: "Study of ancient dolomitic mortars of the church of Santa María de Zamarce in Navarra (Spain): comparison with simulated standards". Thermochim. Acta, vol. 398 (2003), pp. 107-122. doi: 10.1016/S0040-6031(02)00321-0.

(23) Moropoulou, A.; Bakolas, A.; Bisbikou, K.: "Characterization of ancient, byzantine and later historic mortars by thermal and X-ray diffraction techniques". Thermochim. Acta, vol. 269-270 (1995), pp. 779-795. doi: 10.1016/0040-6031(95)02571-5.

(24) Dorrego, F.; Luxán, M. P.; Sotolongo, R.: "Los trabadillos": Origen, utilización y técnicas de preparación. Actas del II Congreso Nacional de Historia de la Construcción editado por Instituto Juan de Herrera. La Coruña (1998), pp. 145-150.

(25) Genestar, C.; Pons, C.: "Ancient covering plaster mortars from several convents and Islamic and Gothic palaces in Palma de Mallorca (Spain)". J Cult Herit, vol. 4 (2003), pp. 291-298. doi: 10.1016/j.culher.2003.02.001.

(26) Luxán, M. P.; Dorrego, F.; Sotolongo, R.: "Mortars and treatments in the restoration of the Altarpiece of the High Altar of the Pilar Basilica". Mater. Construcc., vol. 50, no 258 (2000), pp. 27-36. 